\title{
Cardiovascular Function Is Altered by Picomole Injections of Glutamate into Rat Medulla
}

\author{
D. O. Nelson, Howard L. Cohen, Jack L. Feldman, ${ }^{a}$ and Donald R. McCrimmon \\ Departments of Physiology, Anesthesia, and Surgery, Northwestern University Medical School, Chicago, Illinois 60611
}

\begin{abstract}
Local neural circuitry in the nucleus tractus solitarius (NTS) involved in cardiovascular control was studied by injecting nanoliter volumes of excitatory amino acids into the structure. Experiments were performed on urethane-anesthetized, artificially ventilated rats. Multibarrel micropipettes were used for pressure ejection of drugs or a dye for marking ejection sites. Ejected volumes, ranging from $200 \mathrm{pl}$ to 25 $\mathrm{nl}$, were directly monitored for every injection. Injections of as little as $\mathbf{2 0 0} \mathrm{fmol}$ of L-glutamate in $\mathbf{2 0 0} \mathrm{pl}$ into the medial and lateral NTS region rostral to the obex elicited marked, site-specific decreases in arterial pressure and heart rate. The majority of these responses were eliminated by blockade of parasympathetic and sympathetic neural outflow. At sites caudal to obex, in the commissural region of the NTS, L-glutamate injections produced marked elevations in heart rate and arterial pressure which were sympathetically mediated. Responses to L-glutamate were attenuated by concurrent injection of glutamic acid diethyl ester and DL-2-amino-4-phosphonobutyrate, or lidocaine. These results indicate a heterogeneity in the spatial organization of brain-stem circuitry underlying cardiovascular control that has not been previously described.
\end{abstract}

The nucleus tractus solitarius (NTS) is the primary site of termination of afferent nerve fibers arising from arterial and cardiopulmonary mechanoreceptors (Crill and Reis, 1968; Murra and Reis, 1969; Seller and Illert, 1969; Jordan and Spyer, 1977; Ciriello and Calaresu, 1981). Neurons in the NTS integrate these and other circulatory related afferent signals and are important in the production of appropriate patterns of cardiovascular, pulmonary, and other homeostatic responses (Spyer, 1981). Lesions within this structure have far more severe influences than sinoaortic denervation on cardiovascular function. In the latter situation, arterial pressure shows only lability (Cowley et al., 1980) compared with fulminating hypertension with pulmonary edema and chronically maintained hypertension with the former (Doba and Reis, 1973; Dejong and Palkovits, 1976; Talman et al., 1981). The rich innervation of the NTS by visceral afferents and numerous other CNS structures coupled with multiple ef-

Received Aug. 22, 1986; revised Oct. 2, 1987; accepted Oct. 5, 1987

The technical assistance of Carol A. Graham and Marianne Otto is gratefully acknowledged. This work was supported by NIH Grants HL-29033 and NS-21036 and the Schweppe Foundation.

Correspondence should be addressed to Dr. Douglas O. Nelson, Department of Physiology, Northwestern University Medical School, 303 East Chicago Avenue, Chicago, IL 60611 .

${ }^{\circ}$ Present address: Department of Kinesiology, University of California Los Angeles, 405 Hilgard Avenue, Los Angeles, CA 90024.

Copyright (C) 1988 Society for Neuroscience $0270-6474 / 88 / 051684-10 \$ 02.00 / 0$ ferent paths provides the neuroanatomical substrate for integration and modulation of cardiovascular responses to mechanoreceptor afferent signals. Thus, the NTS must function as more than a simple relay nucleus.

The neuronal integration within the NTS requires information transfer between neurons in a complex network. The detailed nuclear substructure and microanatomy of the NTS suggests that small aggregates of neurons may act as functional units. Yet, despite a growing body of neuroanatomical literature indicating a complex substructure within the NTS (Kalia and Mesulum, 1980; Paxinos and Watson, 1986; Kalia et al., 1984; Kalia and Fuxe, 1985), this structure is often considered to possess fairly homogeneous function with respect to its role in cardiovascular control. In this paper we describe an approach that indicates a functional heterogeneity in the NTS. Utilizing site-specific delivery of subpicomolar amounts of excitatory amino acids in nanoliter volumes, we present evidence for a complex regional organization underlying NTS integration and control of the circulation.

\section{Materials and Methods}

Experiments were performed in 36 urethane $(1 \mathrm{gm} / \mathrm{kg})$-anesthetized rats weighing 300-400 gm. Polyethylene catheters were inserted into the left femoral artery and vein. The arterial catheter was connected via a pressure transducer (Statham) to a polygraph for continuous recording of pulsatile and mean arterial pressures (MAP). Heart rate was determined by an integrating rate meter (Winston Electronics) driven by the lead II ECG signal. In some cases, tracheal cannulas were inserted and animals paralyzed with gallamine trethiodide $(1 \mathrm{mg} / \mathrm{kg})$ and mechanically ventilated. The animals were placed in a stereotaxic frame with the head inclined downward by $45^{\circ}$, and an occipital craniotomy was performed. The caudal portion of the fourth ventricle was exposed by incising the dura and arachnoid and, when necessary, by gentle retraction of the cerebellum with a blunt glass probe.

Two- and three-barrel glass micropipettes were constructed with total tip diameters of $5-20 \mu \mathrm{m}$. Barrels contained 1 or $10 \mathrm{~mm}$ L-glutamate (Glu), a mixture of $100 \mathrm{~mm}$ glutamic acid diethyl ester (GDEE) and 10 mM DL-2-amino-4-phosphonobutyrate (APB), 4\% lidocaine hydrochloride, $2 \%$ pontamine sky blue, or $150 \mathrm{~mm} \mathrm{NaCl}$. Drugs were prepared in $150 \mathrm{mM} \mathrm{NaCl}$ and $\mathrm{pH}$ adjusted to 7.4 with $1 \mathrm{~N} \mathrm{NaOH}$; pontamine was prepared in $0.5 \mathrm{~m}$ sodium acetate. At closely spaced sites within the NTS, measured volumes of drugs were injected with short-duration $(10-500 \mathrm{msec})$ pressure $(0.1-7.0$ atmospheres) pulses. Ejectate volumes were measured directly by monitoring the movement of the fluid meniscus in a pipette barrel with the aid of a $150 \times$ compound microscope equipped with a fine reticule as described previously (McCrimmon et al., 1986). Direct monitoring of drug volume was imperative, as flow resistance of the electrode tip could change significantly following small $(<100 \mu \mathrm{m})$ displacements of the electrode. In experiments involving electrical stimulation of the NTS region, one barrel of a multibarrel micropipettc was filled with $150 \mathrm{~mm} \mathrm{NaCl}$ in $1 \%$ agar. For current passage we used a silver-silver chloride wire inserted into the agar solution and connected to a constant-current stimulus isolation unit connected to a stimulator. Current passed was calculated from the voltage drop across a series resistor in the ground path. 

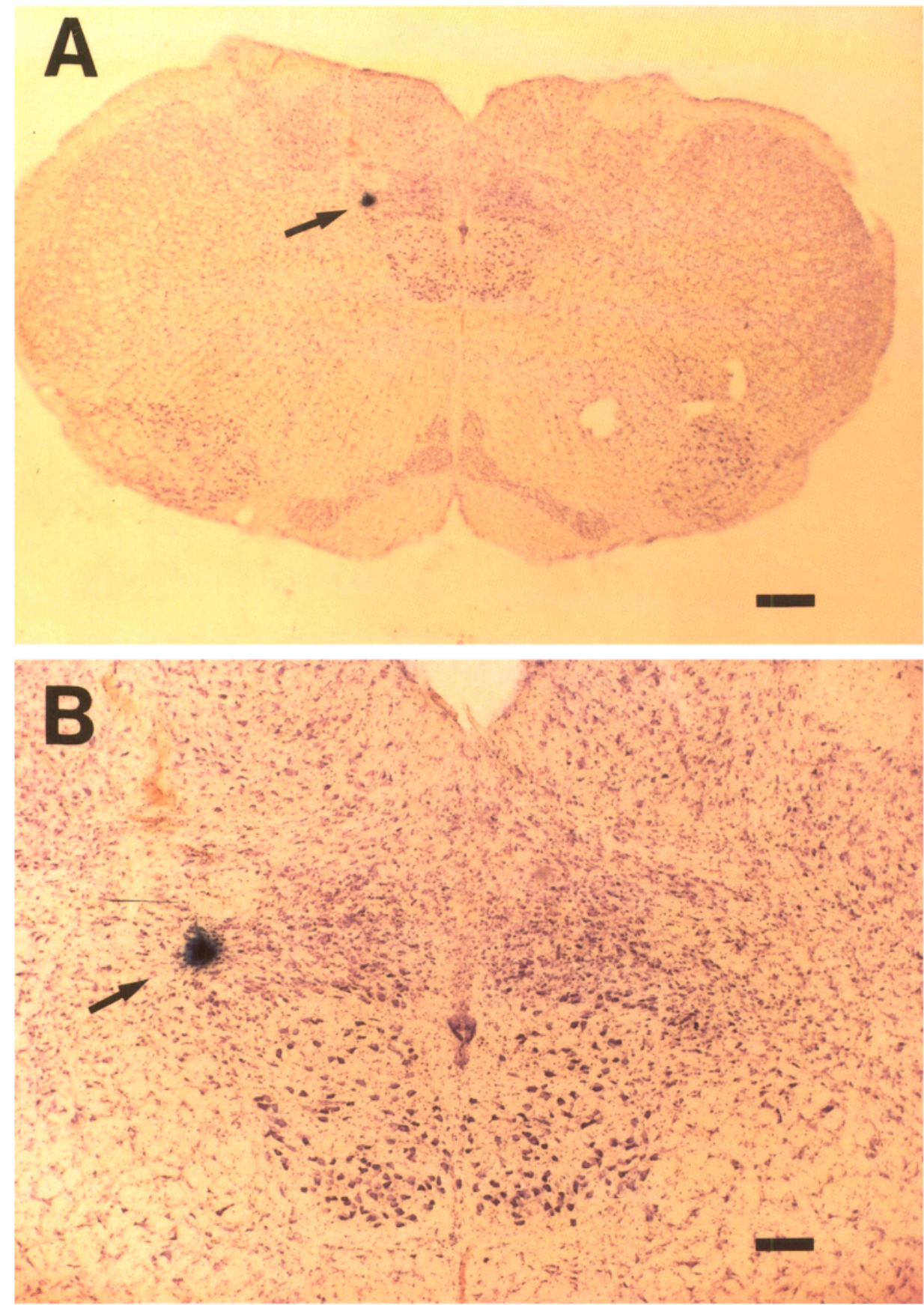

Figure 1. Photomicrograph of a Nisslcounterstained coronal brain-stem section at low $(A)$ and high $(B)$ power illustrating a typical iontophoretically applied dye spot (arrow) marking the bottom of a penetration tract through the lateral aspect of the commissural region of the NTS. Scale bar, $400 \mu \mathrm{m}$ $(A)$ and $100 \mu \mathrm{m}(B)$.
Mapping studies were carried out using 2- or 3-barrel micropipettes containing 1 or $10 \mathrm{~mm}$ Glu, pontamine sky blue, and either saline or an antagonist. The micropipette was positioned on the surface of the dorsal medulla and driven into the brain in 100-200 $\mu$ m steps. Penetration tracks were referenced relative to the predetermined coordinates of the obex and separated by $100-200 \mu \mathrm{m}$. A region extending from 1.5 $\mathrm{mm}$ rostral to $1 \mathrm{~mm}$ caudal to the obex, from midline to $1.2 \mathrm{~mm}$ laterial to midline, and from the dorsal medullary surface to approximately 1.4 $\mathrm{mm}$ ventral was mapped. This region was chosen because it encompasses most of the NTS complex in which cardiovascular-related afferents are believed to terminate (Berger, 1979; Panneton and Loewy, 1980). At each site, $0.2-20 \mathrm{nl}$ of Glu was injected, and the arterial pressure and heart rate responses were recorded on the polygraph and analog tape. In subsequent experiments, additional electrode barrels contained APB and GDEE or lidocaine. At each site, volumes of 150 $\mathrm{mm} \mathrm{NaCl}$ equal to the largest volume of Glu were injected to control for possible nonspecific volume effects. At the site of deepest penetration in an electrode tract, pontamine sky blue dye was iontophoretically injected ( $20 \mu \mathrm{A} \mathrm{DC}$ for $10 \mathrm{~min})$ to mark the injection site precisely.

Using this protocol, we determined at selected sites (1) the dose (volume and concentration) dependence of blood pressure and heart rate responses, (2) the susceptibility of these responses to antagonism by known excitatory amino acid receptor blocking agents, and (3) the region from which a particular response was elicited, by dye injection and histological reconstruction. In some animals, the effects of parasympathetic blockade with atropine methyl nitrate $(1.5 \mathrm{mg} / \mathrm{kg}$, IV) followed by peripheral sympathetic blockade using phentolamine $(1 \mathrm{mg} / \mathrm{kg} \mathrm{IV})$ on Glu-elicited depressor responses were measured. Similarly, in other animals, the effects of administration of propranolol $(0.5 \mathrm{mg} / \mathrm{kg}$, IV) followed by phentolamine and atropine methyl nitrate on Glu-elicited pressor responses were examined. Receptor blockade of peripheral $\alpha$-receptors was tested by intravenous administration of phenylephrine (100 
Figure 2. Dose-dependent decreases in arterial pressure and heart rate produced by injections of Glu at a single site in the NTS. $A$, Reconstruction of injection site (dot) located approximately $1100 \mu \mathrm{m}$ rostral to obex in the medial NTS and $750 \mu \mathrm{m}$ below the brain surface. $B$, Heart rate $(H R, \mathrm{bpm})$ and arterial pressure $(B P, \mathrm{~mm} \mathrm{Hg}) \mathrm{re}-$ sponses to nanoliter injections of $10 \mathrm{~mm}$ Glu. Arrows and numbers below arrows indicate time and volume (in nanoliters) of injections. Notc the rapid-onsct decrease in arterial pressure, but not heart rate, which occurred with $250 \mathrm{pl}$ (2.5 pmol) of Glu. C, Dose-response curve depicting decreases in arterial pressure and heart rate as a function of Glu injection volume for this site. Threshold of the heart rate response was approximately $750 \mathrm{pl}(7.5 \mathrm{pmol})$ of Glu, while the threshold volume for arterial pressure effects was below 250 pl $(2.5 \mathrm{pmol}) . A P$, area postrema; $X$ dorsal motor nucleus of the vagus; $X I I$, hypoglossal nucleus; INTS, lateral nucleus tractus solitarius; $m N T S$, medial nucleus tractus solitarius; $t s$, tractus solitarius.
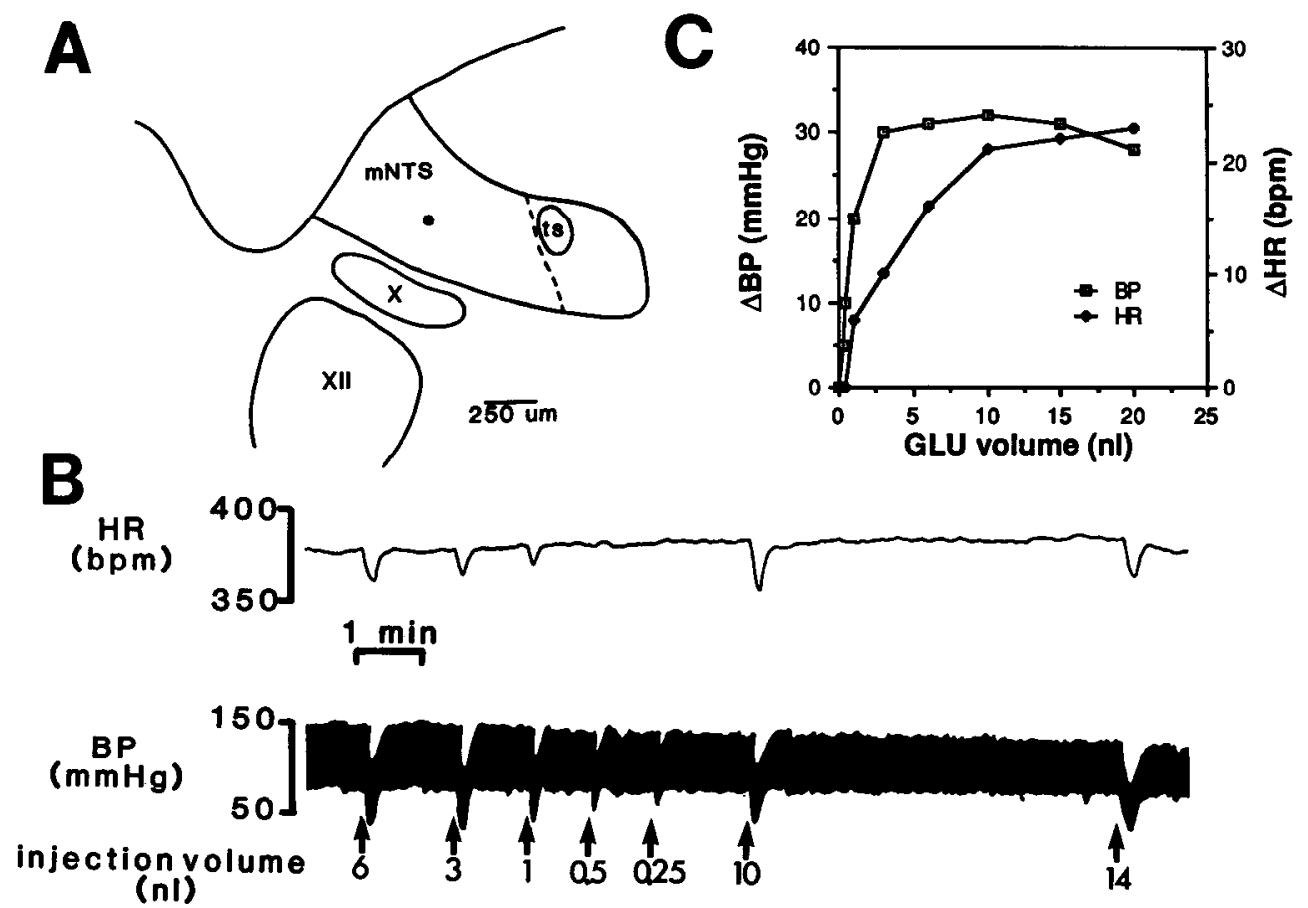

$\mu \mathrm{g} / \mathrm{kg}$, IV). Lack of reflex bradycardia to arterial pressure elevation by $>50 \mathrm{~mm} \mathrm{Hg}$ with phenylephrine was used to indicate parasympathetic blockade. Cardiac $\beta$-receptor blockade was not routinely tested, but the dose used is a generally accepted level for complete blockade.

At the end of each experiment, the animal was deeply anesthetized and perfused transcardially with buffered saline followed by $10 \%$ formalin. The brain stem was removed for subsequent histological reconstruction of the injection sites using standard procedures. Stereotaxic coordinates were compared and corrected against injection sites determined by iontophoretic dye injection.

The significance of changes in arterial pressure and heart rate resulting from microinjections into the brain stem was determined by Student's $t$ test (Siegel, 1956).

\section{Results}

Injections of small volumes $(0.2-20 \mathrm{nl})$ of Glu into discrete regions of the NTS elicited site-specific, dose-dependent, highly reproducible increases or decreases in arterial pressure and heart rate. Potent depressor, decelerator responses were obtained at sites in the NTS extending from the level of the obex rostral approximately $1500 \mu \mathrm{m}$. Figure 1 shows a typical photomicrograph of a dye spot at 2 magnifications used to reconstruct a single tract through the commissural region of the NTS. Dye deposited by iontophoresis produced a high-density confined mark that allowed accurate determination of the microelectrode tip and reconstruction of the other injection sites in the tract using stereotaxic coordinates.

A typical dose-dependent decrease in arterial pressure and heart rate is shown in Figure 2. In response to injections of less than $1 \mathrm{nl}$ (meas'ired directly) of $10 \mathrm{~mm}$ Glu ( $<10 \mathrm{pmol}$ ), marked transient decreases in arterial pressure were elicited with no discernible change in heart rate. Thus, $2.5 \mathrm{pmol}$ Glu produced a decrease in arterial pressure of $12 \mathrm{~mm} \mathrm{Hg}$. Increasing the injection volume above $1 \mathrm{nl}$ produced greater decreases in arterial pressure with accompanying bradycardia. In this example, the peak decrease in arterial pressure was maximal with a $3 \mathrm{nl}$ injection volume of Glu. Maximal bradycardia occurred at a larger injection volume. Figure $2 C$ plots the change in arterial pressure and heart rate against injection volume. Separation of both the threshold and maximal response of arterial pressure and heart rate was common at most sites within the medial NTS. A further increase in injection volume above the level at which a maximal response was obtained usually prolonged the recovery time of the response, as can be seen in Figure 2. Both the heart rate and arterial pressure responses tended to recover gradually to their base levels with no apparent overshoot. At some sites, a slight overshoot of heart rate during recovery was obscrved.

The site dependence of depressor and decelerator responses elicited in the NTS, particularly the medial division, is illustrated in Figure 3. The micropipette tip was advanced into the medial NTS in $200 \mu \mathrm{m}$ increments as shown. At a depth of 250 $\mu \mathrm{m}$ below the medullary surface, neither heart rate nor arterial pressure changes could be elicited with volumes less than $15 \mathrm{nl}$. At a depth of $500 \mu \mathrm{m}$ both decreases in arterial pressure and heart rate were elicited with volumes of less than $10 \mathrm{nl}$. Very low doses at this site produced readily detectable bradycardia responses accompanied by little or no depressor effect. With further advancement of the electrode tip, the threshold volume to elicit arterial pressure changes decreased (Fig. $3 C$ ). Significant depressor effects with no accompanying heart rate changes could be elicited with volumes on the order of $1 \mathrm{nl}$ at a depth of 700 $\mu \mathrm{m}$ (site 3), in contrast to the effects of Glu observed at a depth of $500 \mu \mathrm{m}$. Sensitivity of the heart rate responses increased slightly (Fig. 3, site 3). Advancement of the electrode tip 200 $\mu \mathrm{m}$ to site 4 dramatically raised the threshold to elicit both cardiovascular responses. This type of depth profile was typical for the NTS sites rostral to the obex. Heart rate effects were usually detected with small injection volumes at superficial sites in the medial NTS and tended to exhibit a constant threshold and sensitivity over a wide range of depth $(300-600 \mu \mathrm{m})$. Arterial pressure responses were elicited at more ventral sites and generally peaked sharply at approximately $700 \mu \mathrm{m}$ below the surface. This result is illustrated for this electrode track in Figure 


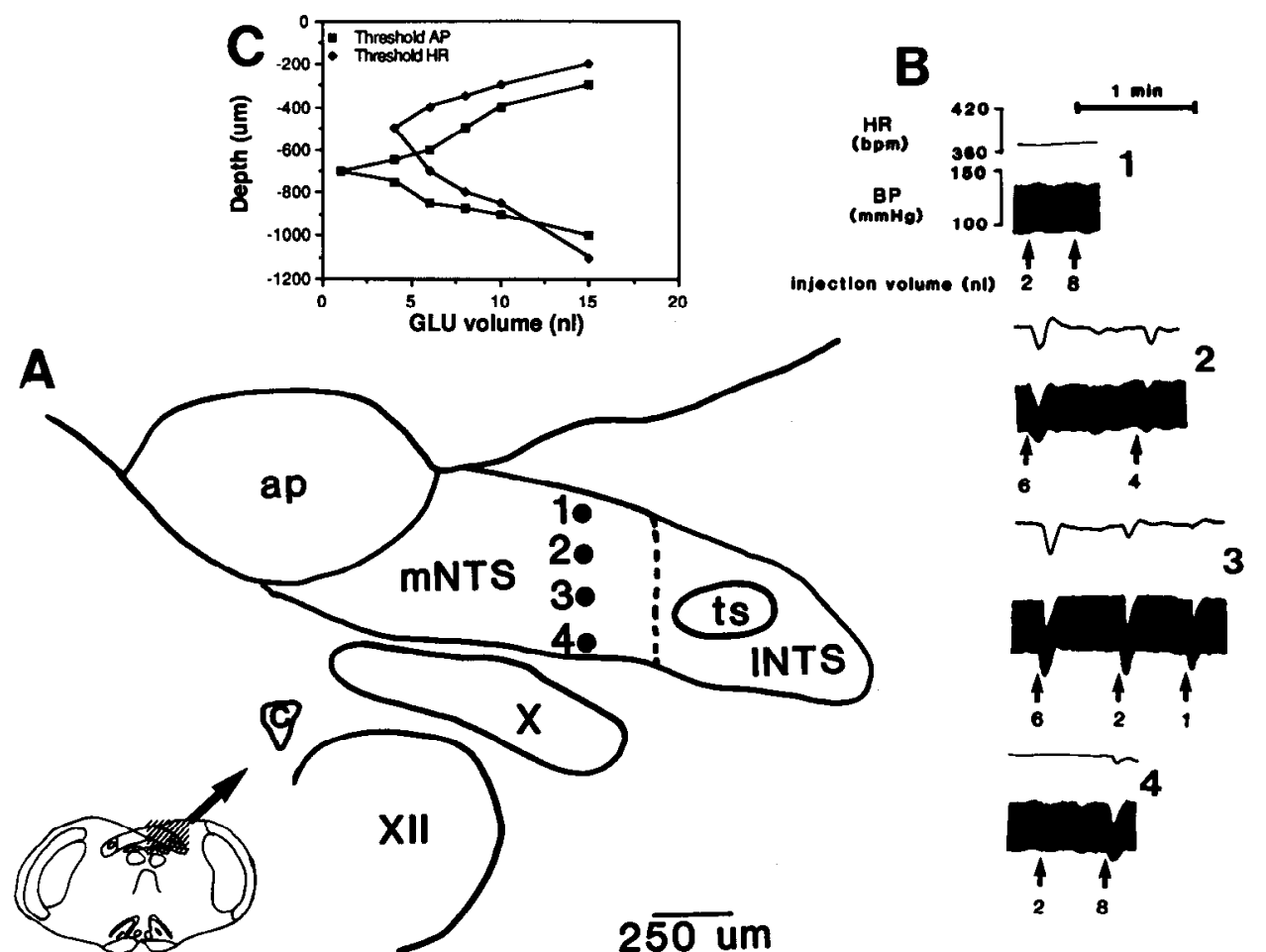

Figure 3. Depth dependence of decreases in arterial pressure and heart rate to Glu injection during a single microelectrode track through the NTS. $A$, Reconstruction of 4 injection sites located $200 \mu \mathrm{m}$ apart in the medial NTS approximately $200 \mu \mathrm{m}$ rostral to obex at the level of the area postrema. Site 1 is $250 \mu \mathrm{m}$ below the medullary surface. $B$, Examples of arterial pressure $(B P, \mathrm{~mm} \mathrm{Hg})$ and heart rate $(H R, \mathrm{bpm})$ responses to injections of $10 \mathrm{~mm}$ Glu for each of the corresponding 4 sites shown in A. Arrows indicate time of injection. Injectate volume is shown below each arrow in nanoliters. Note the increasing sensitivity to Glu with depth. $C$, Depth-threshold profile indicating the volume of $10 \mathrm{~mm}$ Glu needed to produce a detectable decrease in arterial pressure $(A P)$ or heart rate $(H R)$ at various depths below the brain surface. $X$, dorsal motor nucleus of the vagus; $X I I$, hypoglossal nucleus; $m N T S$, medial nucleus tractus solitarius; $t s$, tractus solitarius.

$3 C$, which plots the volume of $10 \mathrm{~mm}$ Glu needed to elicit a detectable change in arterial pressure or heart rate as a function of depth below the brain surface. Each point represents the average of at least 3 injections.

Injections into sites near the obex in the commissural region of the NTS and extending $1000 \mu \mathrm{m}$ caudal produced graded pressor and cardioaccelerator responses. Dose-dependent increases in heart rate and blood pressure to injection volumes ranging from 2 to $20 \mathrm{nl}$ of $1 \mathrm{~mm}$ Glu (2-20 pmol) are shown in Figure 4. Responses to a given injection volume were highly reproducible. At the injection site in Figure 3, increasing the volume above $3 \mathrm{nl}$ did not significantly increase the peak change in arterial pressure or heart rate but did prolong the duration of each response. The large increases in arterial pressure elicited at sites in the commissural region of the NTS did not produce reflex bradycardia responses even with pressor response durations lasting $30 \mathrm{sec}$. Pressor responses accompanied by bradycardia were not observed at any site in this region. The dose dependence of the arterial pressure and heart rate responses is illustrated in Figure $4 C$. Typically, arterial pressure and heart rate responses at a single injection site showed a pronounced sensitivity to Glu that plateaued at injection volumes similar to those shown in Figure 3.

The depth dependence of pressor sites in the commissural region of the NTS is illustrated in Figure 5 . Note that at site number 1 , approximately $200 \mu \mathrm{m}$ below the dorsal brain-stem surface, large volumes of Glu ( $16 \mathrm{nl})$ elicited significant increases in arterial pressure and heart rate, while a small volume $(1 \mathrm{nl})$ was ineffective. As the electrode tip was advanced in $150 \mu \mathrm{m}$ increments, the volume of Glu needed to produce a pressor response diminished. The lowest threshold site was approximately $400-500 \mu \mathrm{m}$ below the medullary surface between sites 2 and 3. Injection volumes of Glu as small as $200 \mathrm{pl}(2 \mathrm{pmol})$ elicited $15 \mathrm{~mm} \mathrm{Hg}$ pressor responses at this depth with a small accompanying tachycardia $(<5 \mathrm{bpm})$. A more pronounced tachycardia was produced with greater injection volumes. Also note that, compared to the heart rate changes elicited at the site shown in Figure 4, the effects on heart rate produced at this site were significantly smaller for a given injection volume. As the electrode tip was advanced further, the cardiovascular responses to Glu injection diminished and finally disappeared. Figure $5 C$ illustrates the changes in threshold volume to elicit arterial and heart rate changes as a function of depth for this electrode track. Since $200 \mathrm{pl}$ represents the effective resolution of our pressure injection system, we were unable to precisely determine the threshold at this site. The threshold-depth profile shown in Figure $5 C$ indicates that the heart rate and arterial pressure effects of Glu in this electrode track were not readily separated by adjustment of electrode depth. The coupling of the effect of Glu on heart rate and arterial pressure was common to all sites examined in the commissural region.

At 17 scattered sites in the NTS where Glu injection produced robust cardiovascular changes, blockade of the responses to Glu was attempted using prior or simultaneous microinjections of a combination of $10 \mathrm{~mm}$ APB and $100 \mathrm{~mm}$ GDEE from a separate electrode barrel. The results of these experiments at 2 sites in the medial NTS are illustrated in Figure 6. Successive injections of the antagonists gradually decreased and finally eliminated the depressor and bradycardia effects produced by $2 \mathrm{nl}$ injections of $10 \mathrm{~mm}$ Glu (Fig. 6A). At a different site, 30 $\mathrm{nl}$ injections of APB and GDEE completely eliminated the cardiovascular responses to Glu injections (Fig. $6 B$ ). Following a $10 \mathrm{~min}$ recovery period, the effects of Glu returned to near control levels. A 10-15 min time course of recovery was typical. Also note that injection of the antagonist mixture did not produce any detectable change in resting arterial pressure or heart rate. Even with injection volumes of $100 \mathrm{nl}$, no changes in basal heart rate or arterial pressure at any of the sites examined were 
A

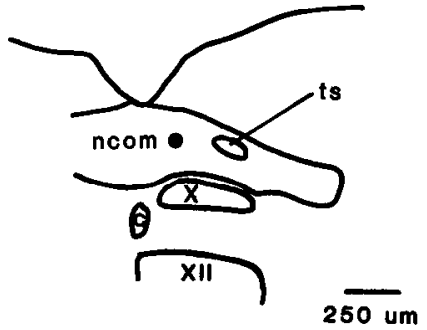

Figure 4. Dose-dependent increases in arterial pressure and heart rate produced by injections of Glu at a single site in the caudal NTS. $A$, Reconstruction of the injection site (dot) located $500 \mu \mathrm{m}$ below the medullary surface at a level approximately $600 \mu \mathrm{m}$ caudal to obex in the commissural region. $B$, Heart rate $(H R, \mathrm{bpm})$ and arterial pressure $(B P, \mathrm{~mm} \mathrm{Hg})$ responses to nanoliter injections of $10 \mathrm{~mm}$ Glu. Arrows and associated numbers indicate injection occurrence and volume in nanoliters. Dose-response curves plotting increases in arterial pressure $(B P)$ and heart rate $(H R)$ for several injection volumes of $10 \mathrm{~mm} \mathrm{Glu}$. $X$, dorsal motor nucleus of the vagus; $X I I$, hypoglossal nucleus; $C$, central canal; ncom, commissural nucleus of the solitary nucleus; $t s$, tractus solitarius.
8

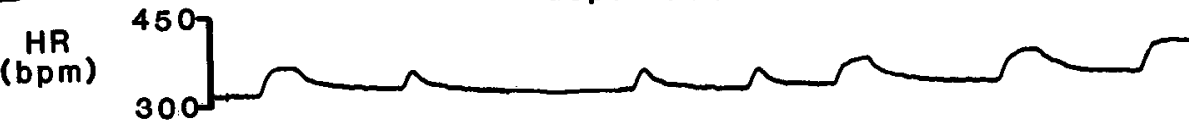

detected following injection of antagonists. APB and GDEE administered in similar volumes were also equally effective in eliminating pressor-cardioaccelerator responses to Glu injection. We did not note any differences in antagonist effects between depressor and pressor sites. Figure $6 C$ illustrates the blockade of Glu-induced changes in arterial pressor and heart rate following injection of $4 \%$ lidocaine. Again, no detectable changes in basal levels of either parameter were noted. Responses to Glu injection typically recovered from lidocaine blockade within 15 min.
Figure 5. Depth dependence of arterial pressure and heart rate responses to microinjections of Glu into the caudal NTS. $A$, Reconstruction of electrode tract through commissural region of the NTS approximately $600 \mu \mathrm{m}$ caudal to the obex. Six injection sites are shown, each $150 \mu \mathrm{m}$ apart. $B$, Six sets of traces corresponding to each of the 6 sites in $A$ arc shown to illustrate the arterial pressure $(B P, \mathrm{~mm} \mathrm{Hg})$ and heart rate $(H R, b p m)$ responses to Glu injection. Arrows and the numbers beneath indicate the time and volume (nl) of 10 mM Glu injected, respectively. Note the significant responses to $200 \mathrm{pl}(2 \mathrm{pmol})$ of Glu at sites 2 and 3. $C$, Volume (nl) of $10 \mathrm{~mm}$ Glu needed to elicit a threshold increase in arterial pressure $(A P)$ or heart rate $(H R)$ is plotted as a function of depth below the medullary surface. $X$, dorsal motor nucleus of the vagus; $X I I$, hypoglossal nucleus; $C$, central canal; ncom, commissural region of the NTS; $t s$, tractus solitarius.

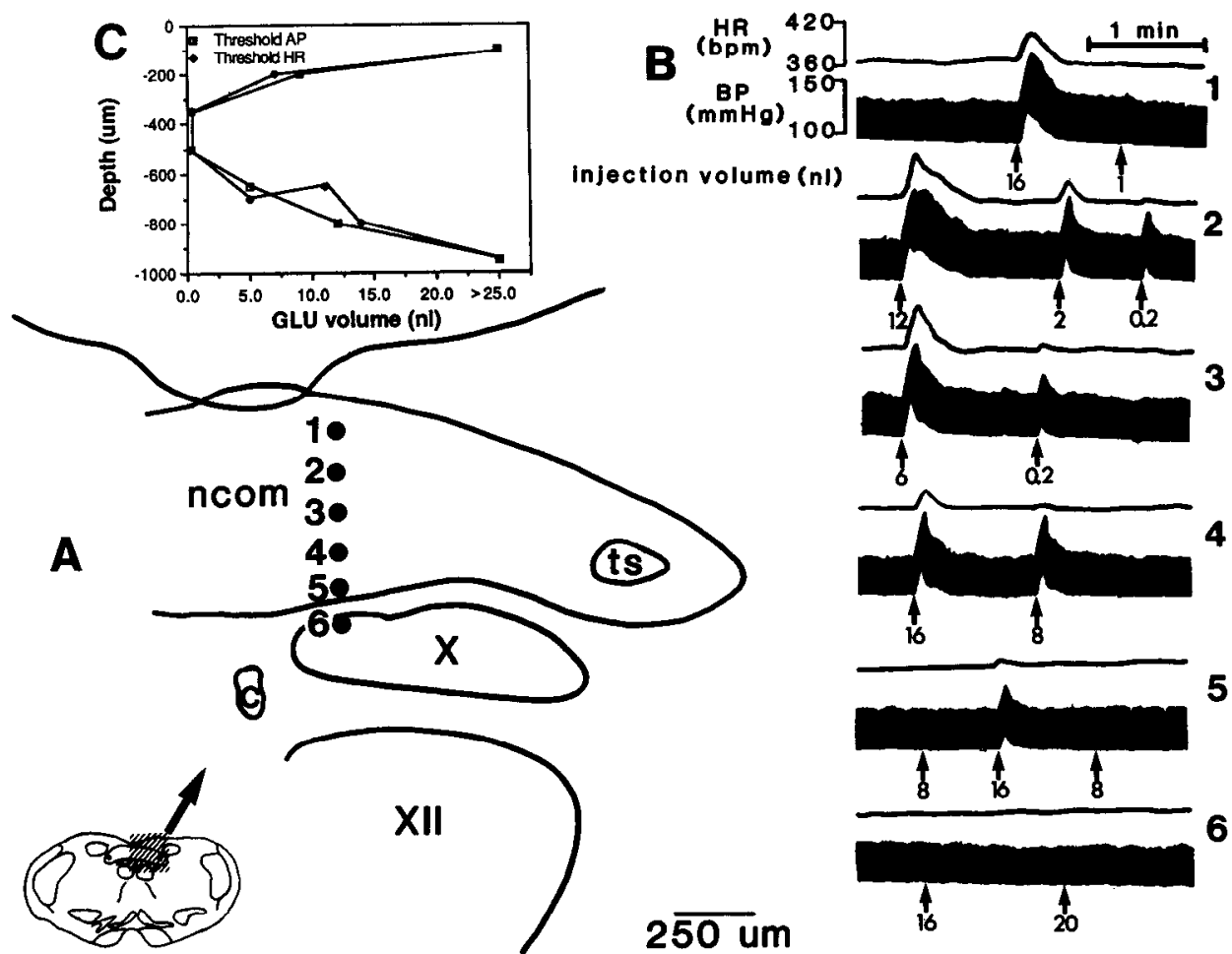


Table 1. Changes in mean arterial pressure $(\Delta \overline{\mathrm{BP}})$ and mean heart rate $(\Delta \overline{\mathrm{HR}})$ elicited by microinjections of $5 \mathrm{nl}$ of $10 \mathrm{~mm}$ Glu before and after drug administration

\begin{tabular}{lcc} 
& \multicolumn{2}{c}{ Response to $5 \mathrm{nl}$ of $10 \mathrm{mM} \mathrm{Glu}$} \\
\cline { 2 - 3 } Sites & $\Delta \overline{\mathrm{BP}}(\mathrm{mm} \mathrm{Hg})$ & $\Delta \overline{\mathrm{HR}}(\mathrm{bpm})$ \\
\hline Depressor-cardiodecelerator & $(n=14)$ & \\
Control & $-37 \pm 7$ & $-29 \pm 4$ \\
After atropine & $-27 \pm 6$ & $-3 \pm 2^{a}$ \\
After phentolamine & $-3 \pm 1^{a}$ & $-2 \pm 2^{a}$ \\
Pressor-cardioaccelerator $(n=10)$ & \\
Control & $49 \pm 12$ & $44 \pm 11$ \\
After propranolol & $33 \pm 9$ & $12 \pm 5^{a}$ \\
After phentolamine & $5 \pm 2^{a}$ & $8 \pm 4^{a}$ \\
After atropinc & $4 \pm 2^{a}$ & $3 \pm 4^{a}$ \\
\hline
\end{tabular}

a $p<0.05$ compared to control values. Changes are expressed as changes from baseline during control, after propranolol, phentolamine, or atropine administration.

At 24 scattered sites along the rostral-caudal extent of the NTS where Glu injection produced cardiovascular changes, intravenous injections of autonomic blocking agents were utilized to ascertain the efferent pathways producing the observed cardiovascular responses (Table 1). Depressor responses were unaffected by intravenous atropine methyl nitrate but were totally eliminated by subsequent intravenous administration of phentolamine. At these sites, atropine reduced the cardiodecelerator effect of $5 \mathrm{nl} \mathrm{Glu}$ injections by $89 \%$. Addition of phentolamine did not further reduce the remaining response. However, at 4 sites located deep in the NTS close to the dorsal motor nucleus of the vagus (DMNV), atropine alone completely eliminated both the depressor and decelerator effects of Glu injections. Control responses to $5 \mathrm{nl}$ of $10 \mathrm{~mm}$ Glu consisted of a $35 \pm 8$ $\mathrm{mm} \mathrm{Hg}$ decrease in mean arterial pressure and a $31 \pm 6 \mathrm{bpm}$ bradycardia. These responses were completely eliminated by atropine treatment. An example of one of the sites is shown in Figure 7. At a depth of $700 \mu \mathrm{m}$ below the dorsal brain-stem surface, a $6 \mathrm{nl}$ injection produced a decreased arterial pressure and bradycardia indistinguishable from those elicited at a depth of $500 \mu \mathrm{m}$. Atropine methyl nitrate administration completely eliminated these effects. Only at sites, such as the one illustrated, near the DMNV within $500 \mu \mathrm{m}$ of the obex did vagal blockade fully attenuate the Glu responses. The responses to Glu at all other sites appeared to include both a sympathetic and cardiac vagal component.

The effect of administration of propranolol followed by phentolamine and then atropine on cardiovascular responses to $5 \mathrm{nl}$ Glu injections was tested at 10 sites where pressor-cardioaccelerator responses were found. Propranolol alone did not significantly affect the change in blood pressure but did significantly reduce (by $70 \%$ ) the cardioaccelcrator effect of a $5 \mathrm{~nL}$ injection of Glu. Addition of phentolamine virtually eliminated the pressor response, reducing it to $10 \pm 4 \%$ of control, but did not further reduce the small remaining cardioaccelerator effect. Subsequent intravenous administration of atropine eliminated the remaining cardioaccelerator responses with no effect on arterial pressure.

Electrical stimulation was compared with the effects of Glu injection at several sites rostral and caudal to obex in the NTS region. Low-intensity electrical stimulation at sites rostral to obex produced potent decreases in heart rate and arterial pres-
A

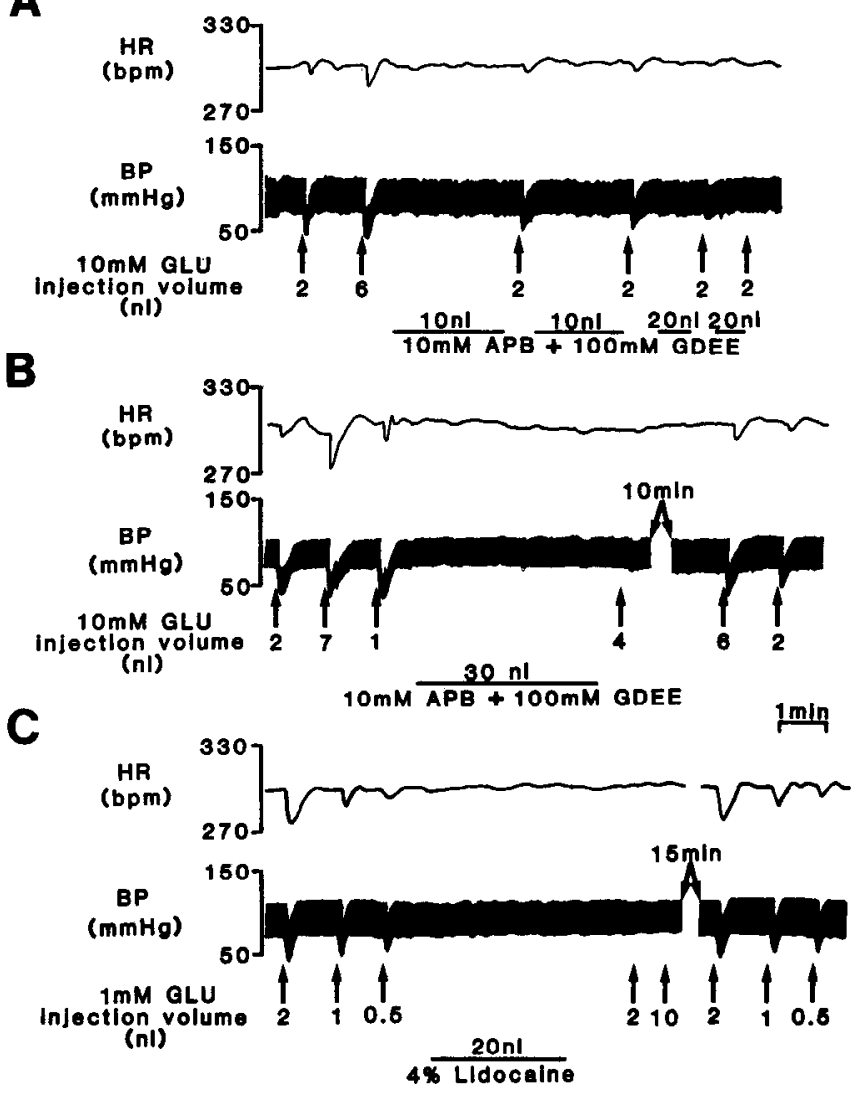

Figure 6. Blockade of Glu effects by microinjection of Glu antagonists or lidocaine in the medial NTS. Shown in each panel are heart rate $(H R$, $\mathrm{bpm})$ and arterial pressure $(B P, \mathrm{~mm} \mathrm{Hg})$ records. Injection time and volume of the indicated Glu concentration are indicated by the arrows and associated numbers. $A$, Successive microinjection of $10 \mathrm{mM}$ APB plus $100 \mathrm{~mm}$ GDEE decreased and eventually eliminated the responses to a $2 \mathrm{nl}(20 \mathrm{pmol})$ injection of Glu. The antagonist mixture was slowly micropressure-injected from the same electrode as indicated by the horizontal bars. $B$, Microinjection of $30 \mathrm{nl}$ of antagonist mixture during the time indicated completely eliminated the response to Glu. Note that following a $10 \mathrm{~min}$ recovery period the Glu response returned. $C$, Blockade of Glu responses by a $20 \mathrm{nl}$ injection of $4 \%$ lidocaine. Recovery was complete after a $15 \mathrm{~min}$ period.

sure. Figure $8 A$ shows the similarity of responses to Glu injections and electrical stimulation $(25 \mathrm{~Hz}, 15 \mu \mathrm{A}$, constant current). Qualitatively similar effects on arterial pressure and heart rate were observed in response to injections of small volumes of 10 $\mathrm{mm}$ Glu and direct electrical stimulation. Heart rate responses generally were more rapid in onset and greater in magnitude with electrical stimulation than with Glu injection. Both the Glu and electrically evoked responses shown in Figure $8 A$ were subsequently eliminated by combined atropinc and phentolamine administration. Figure $8, B-D$, contrasts the effects of Glu and direct electrical stimulation at a site caudal to obex in the commissural region. At this site, small injections of Glu produced pressor responses accompanied by tachycardia (Fig. $8 B$ ). Electrical stimulation, however, produced a prominent decrease in arterial pressure and heart rate. Intravenous administration of propranolul significantly reduced the pressure-tachycardia response to Glu (Fig. $8 \mathrm{C}$ ). In contrast, the effects of electrical stimulation persisted and were slightly enhanced. Phentolamine administration (Fig. $8 D$ ) eliminated the arterial pressure re- 
Figure 7. Atropine sensitivity of response to Glu microinjection into medial NTS near vagal complex. $A$, Reconstruction of 3 injection sites traversing the medial NTS at a level approximately $200 \mu \mathrm{m}$ caudal to the obex. Note the relative proximity of the third site to the dorsal motor nucleus of the vagus. $B$, Arterial pressure ( $B P$, $\mathrm{mm} \mathrm{Hg})$ and heart rate $(H R$, bpm) responses to microinjection of $1 \mathrm{~mm}$ Glu into each of the 3 sites. Note that responses are detectable with a volume of $250 \mathrm{pl}(250 \mathrm{fmol})$. Advancement of the electrode tip from 500 to $700 \mu \mathrm{m}$ below the surface did not significantly alter the responses to $6 \mathrm{nl}$ of Glu. Intravenous administration of atropine methyl nitrate completely eliminated responses to Glu.
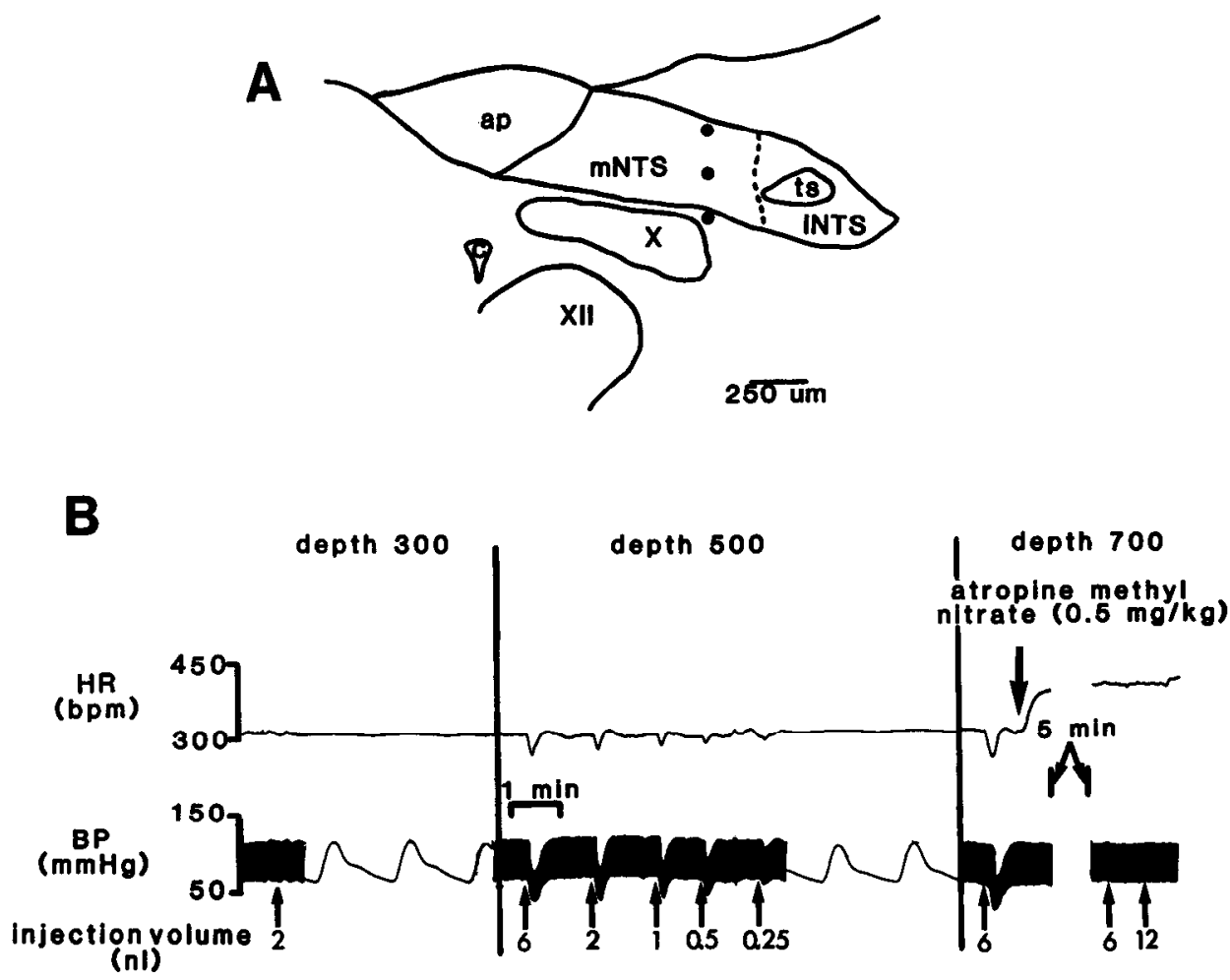

A
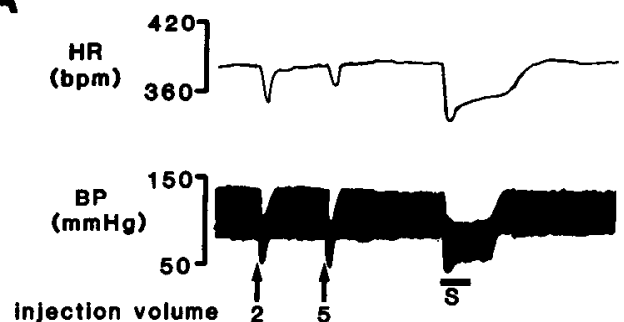

(nl)

B

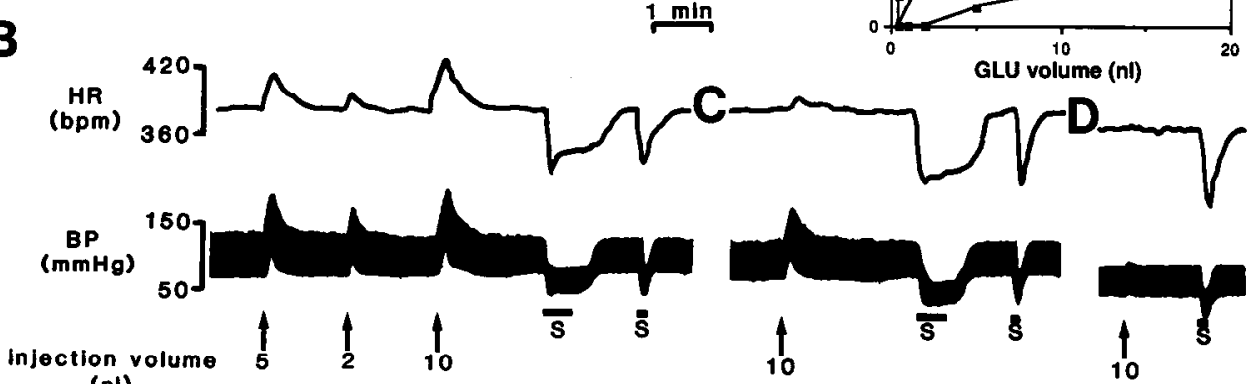

Figure 8. Comparison of electrical stimulation and Glu injection in the NTS. $A$, Decreases in arterial pressure $(B P, \mathrm{~mm} H g)$ and heart rate $(H R$, bpm) produced by microinjections of $10 \mathrm{~mm}$ Glu into a site located $700 \mu \mathrm{m}$ rostral to obex in the medial NTS. Brief electrical stimulation $(S$, 25 $\mathrm{Hz}, 15 \mu \mathrm{A}, 0.5 \mathrm{msec}$ pulse duration) also produced a decrease in arterial pressure and bradycardia. Arrows indicate injection times. Injection volume in nanoliters is indicated below each arrow. $B$, Increases in arterial pressure and heart rate produced by 10 mM Glu microinjection into a site 450 $\mu \mathrm{m}$ caudal to obex. As in $A$, brief electrical stimulation $(S)$ using the same stimulus parameters produced a prominent depressor and bradycardia response. $C$, Partial elimination of the responses to Glu following propranolol administration but with no effect on the responses to electrical stimulation. $D$, Following phentolamine treatment the effects of Glu were nearly eliminated. The response to electrical stimulation persisted. $E$, Dose-response curves plotting increases in arterial pressure and heart rate $(H R, \mathrm{bpm})$ as a function of Glu injection volume. In each graph the responses during control, following propranolol treatment, and following phentolamine administration are shown. 
sponse to Glu but did not affect the response to electrical stimulation. Similar results were observed at additional sites (4 of 6 tested) in the commissurral region. Figure $8 E$ shows curves relating changes in heart rate and arterial pressure for a range of Glu injection volumes. Propranolol administration virtually eliminated the heart rate response and reduced the arterial pressure response to Glu. Subsequent phentolamine administration significantly $(p<0.05)$ reduced the remaining arterial pressure response.

The extent of the NTS region examined and the effects of Glu injection in this study are summarized for 6 animals in Figure 9. Decreases in arterial pressure and heart rate were observed in a region extending from approximately the level of the obex rostral $1500 \mu \mathrm{m}$. Within this region at sites medial to the tractus solitarius Glu injection produced dose-dependent decreases in both heart rate and arterial pressure, although these effects could be separated by depth and injection dosage. The lowest threshold for heart rate effects was at more superficial depths $(\approx 500$ $700 \mu \mathrm{m})$ than for arterial pressure effects $(\approx 700-900 \mu \mathrm{m})$. At lateral sites in the region rostral to the obex, Glu effects were confined to changes in arterial pressure if the injection volumes were kept bclow $10 \mathrm{nl}$ Above this volume heart rate effects were also observed. Responses to Glu volumes of $20 \mathrm{nl}$ or less were absent at more rostral sites in the NTS. In the commissural region of the NTS, beginning approximately $100 \mu \mathrm{m}$ caudal to the obex and extending 500-600 $\mu \mathrm{m}$ lateral to midline, Glu injections produced increases in arterial pressure and heart rate that could not easily be separated by alteration of injection volume or electrode position.

\section{Discussion}

We have observed that injections of as little as $200 \mathrm{fmol}$ (in 200 pl) of an excitatory amino acid into a discrete region of the NTS can elicit marked changes in arterial pressure and heart rate. At most sites, the alterations in cardiovascular function are the result of changes in both sympathetic and parasympathetic outflow. A striking finding, in light of previous studies, is that markcd pressor and cardioaccelerator responses, in addition to depressor and cardiodecelerator effects, can be elicited from sites within the NTS comples, particularly in the commissural region. This is an interesting finding considering that (1) this region receives dense primary baroreceptor afferent innervation (Berger, 1979; Panneton and Loewy, 1980; Ciriello and Calaresu, 1981; Spyer et al., 1984); (2) electrical stimulation within this area produces depressor and bradycardia effects (Crill and Reis, 1968; Dejong et al., 1977); and (3) single baroreceptor fibers often show rather extensive arborization and may terminate in several subdivisions of the NTS complex (Spyer et al., 1984). The observation of pressor responses elicited from the NTS complex raises important questions about afferent processing and the function of intrinsic neurons. Moreover, it indicates that the NTS functions as more than a simple relay of baroreceptor information. It is possible that the pressor responses observed in the commissural region are due to selective activation of neurons involved in reflexes other than the baroreflex, such as the chemoreceptor reflex. More recent anatomical studies in rat (Ciriello, 1983) and cat (Spyer et al., 1984) demonstrate that this area receives significantly fewer baroreceptor afferents than more rostral and lateral regions of the NTS. Further, the commissural region appears to receive a predominance of chemoreceptor input. The results of our study cannot differentiate

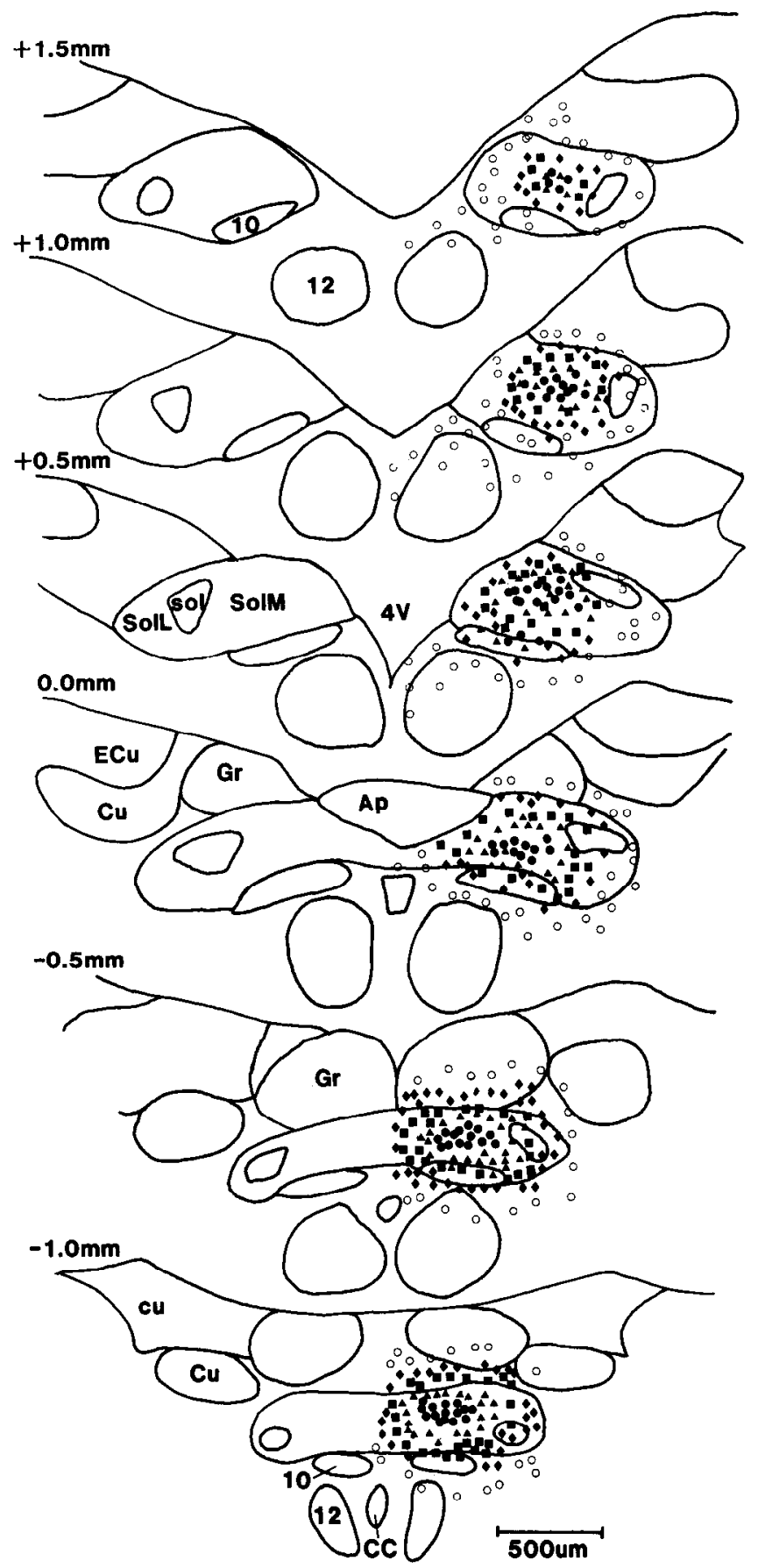

Figure 9. Schematic representation of injection sites in the dorsal medulla by threshold volume in 6 animals in which the right NTS region was mapped. Nuclei are labeled on the left side. The number beside each section denotes the rostral-caudal position of each coronal section relative to the obex. Symbols indicate the threshold volume as follows: filled circles, $<0.5 \mathrm{nl}$; triangle, $<1.0 \mathrm{nl}$; squares, $<5.0 \mathrm{nl}$; diamonds, $<20.0 \mathrm{nl}$; open circles, no response with volumes to $20.0 \mathrm{nl}$. Symbols plotted from the obex $(0.0 \mathrm{~mm})$ to $+1.5 \mathrm{~mm}$ rostral indicate depressorcardiodecelerator effects; symbols at -0.5 and $-1.0 \mathrm{~mm}$ represent pressor-cardioaccelerator effects. Abbreviations: $A p$, area postrema; $C C$, central canal; $\mathrm{Cu}$, cuncate nucleus; $\mathrm{cu}$, cuneate fasciculus; $E C u$, external cuneate nucleus; $G r$, gracile nucleus; sol, solitary tract; SolL, lateral nucleus of the solitary tract; SolM, medial nucleus of the solitary tract; $4 \mathrm{~V}$, fourth ventricle; 10 , dorsal motor nucleus vagus; 12 , hypoglossal nucleus. 
between these possibilities; nonetheless, they do support the idea of a functional differentiation of the subnuclei of the NTS.

The effects of intravenous administration of atropine methyl nitrate, propranolol, and phentolamine indicate that activation of small groups of NTS neurons can affect both the parasympatnetic and sympathetic branches of the autonomic nervous system. At virtually all sites in the dorsal NTS, the hemodynamic responses elicited by Glu injection resulted from reciprocal changes in sympathetic and parasympathetic outflow. Data demonstrating the existence of cardiovagal neurons in the DMNV are equivocal and probably species dependent (Spyer, 1981). At least one report has demonstrated DMNV-mediated alteration of heart rate in the rat (Nosaka et al., 1979). Although the present study was not aimed directly at this question, the observation that Glu could elicit responses (at ventral NTS sites near the level of the obex) that were totally abolished by atropine administration does suggests DMNV cardiovagal neurons in the rat. The decreases in heart rate and arterial pressure elicited at these ventral sites were qualitatively similar to the responses observed at dorsal sites at the same rostral-caudal level, yet the latter responses were minimally affected by parasympathetic blockade; this suggests a significant sympathetic component. We postulate that the cardiovascular changes seen at several of the more ventral sites were probably due to direct activation of proximate cardiovagal neurons in the DMNV since atropine eliminated the response. Pressor-cardioaccelerator responses were probably due to a combination of elevation of both peripheral and cardiac sympathetic effects since $\beta$ and $\alpha$-blockade virtually eliminated the observed responses to Glu.

Our observations impact on recent work implicating Glu as the neurotransmitter utilized by baroreceptor afferents. Reis and coworkers (Talman et al., 1980, 1984; Reis et al., 1981) supported this hypothesis with evidence that Glu, when injected into the NTS in large volumes (typically $100 \mathrm{nl}$ ), produces a dose-dependent decrease in both arterial pressure and heart rate. Using similar concentrations but volumes 1-3 orders of magnitude less $(0.2-10 \mathrm{nl})$, we found that, depending upon the precise site of injection, depressor or pressor responses accompanied by small or substantial heart rate changes can be elicited by Glu injection. In addition, several of the injection sites in these previous studies were close to the DMNV, yet no controls for direct vagal activation were included. We found that responses at these sites were severely attenuated following parasympathetic blockade.

The changes in cardiovascular variables due to injections of the excitatory amino acids are likely to result from increased discharge of neurons with glutamate receptors on their somatodendritic membranes, but not on fibers of passage, in the immediate vicinity of the electrode tip (Curtis and Ryall, 1966; Zieglgansberger and Puil, 1973; Goodchild et al., 1982). A mechanism of action via glutamate receptor activation is supported by the ability of selective glutamate receptor blocking agents to antagonize the responses. Nonetheless, several possible sources of artifact must be considered. First, pressure injection artifacts are possible, but they are ruled out by the absence of effects of equivalent or larger volumes of saline or an antagonist. Second, depolarization block is a potential problem (Crawford and Curtis, 1964; Engberg et al., 1979a, b). This is unlikely since a given response could be reproduced 4-5 times in rapid succession with no noticeable decrease in the response. Also, the magnitude of the response was both volume $(0.2-10 \mathrm{nl})$ and concentration (1-10 $\mathrm{mm}$ ) dependent and did not show a biphasic component suggestive of depolarization blockade (Crawford and Curtis, 1964). In addition, blockade of local neuronal discharge by lidocaine did not mimic the effects of Glu.

The effective spread of Glu is difficult to gauge without direct measurement. Several factors such as available extracellular space ( $1 \mathrm{nl}$ equals a sphere approximately $120 \mu \mathrm{m}$ in diameter), the numbers and types of receptors on regional neurons, and pharmacodynamics would all affect drug spread and response. The observation that tip dispacements of $100-300 \mu \mathrm{m}$ could lead to noticeable changes in the cardiovascular response suggests a limited spread. This is also supported by theoretical calculations showing that injectate concentrations fall very rapidly within small distances $(<300 \mu \mathrm{m})$ from the injection site when administered in similar volumes and concentrations as those utilized in this study (Nicholson, 1985). Glutamate inactivation mechanisms, such as active uptake by surrounding neurons or glia, would further reduce the effective spread. We attempted, without success, to demonstrate Glu spread by using volume injections of dye. Because of the minute injection volumes and the necessary use of low concentations of dye to prevent electrode tip clogging, even the mildest histological processing eliminated the dye spots. Dye spread is probably a poor indicator of drug spread because of differences in mobility, charge/mass ratio, uptake, and inactivation mechanisms.

Although we did not carefully compare the effects of Glu injection with electrical microstimulation at every site, the observations we did make are interesting. Low-intensity $(<25 \mu \mathrm{A})$ electrical microstimulation at low frequencies $(5-25 \mathrm{~Hz})$ tended to produce much greater decreases in heart rate than observed with Glu injection in the rostral NTS regions that we explored. Perhaps most striking is the observation that at more caudal sites electrical stimulation often produced blunted or even opposite cardiovascular effects (depressor, deceleration) than observed with Glu injection. These differences are probably attributable to electrical activation of fibers of passage and current spread. Electrical stimulation, but not Glu injection, may have activated fibers from inhibitory neurons which masked the excitatory response to activation of somatodendritic membranes of intrinsic neurons close to the micropipette tip. Electrical stimulation of fibers of passage is a commonly discussed (Ranck, 1975) experimental problem that has produced conflicting results concerning the role of certain nuclei in cardiovascular regulation (Loewy et al., 1979).

The lack of a major effect of Glu antagonists or lidocaine on blood pressure or heart rate suggests several possibilities concerning Glu actions on the neurons producing the hemodynamic responses. Glu excites neurons with somatodendritic membranes in the vicinity of the injection pipette, but these neurons may not fire spontaneously in our experimental preparation. Therefore, local anesthesia or Glu receptor blockade would have no effect. Such neurons may exhibit spontaneous activity and be important in intact animals. A second possibility is that Glu potently excites neurons with low spontaneous discharge rates. Induction of high rates of synchronous discharge in a small group of neurons in the cardiovascular network might produce a noticeable perturbation. However, a small decrease or complete inhibition of discharge induced by Glu receptor blockade or lidocaine in this small group of cells may have little overall effect on the network. Moreover, it may be necessary to have extensive, and perhaps bilateral, blockade to affect cardiovascular fuctions (Talman et al., 1981). A third possibility might involve Glu receptors located on distal dendrites of cells me- 
diating the response. Thus, application of Glu to the distal dendrite would produce depolarization and could cause a quiescent neuron to fire or substantially increase the discharge rate of an active neuron. If there is a limited endogenous release of transmitters activating these excitatory amino acid receptors under our experimental conditions, blockade of responses elicited from distal dendrites by Glu antagonists or lidocaine application may not have a noticeable effect on neuronal discharge rate being generated by excitatory input at other sites on the neuron.

This is the first report demonstrating a functional heterogeneity of intrinsic NTS neurons controlling cardiovascular function. A striking result of this study is that subpicomolar quantities of Glu delivered in nanoliter volumes can produce readily detectable changes in cardiovascular control. Similar results have been obtained in studies on brain-stem neurons controlling breathing (McCrimmon et. al., 1986). The use of nanoliter injection technqies coupled with appropriate drugs and physiological controls could prove to be a useful approach to unraveling the complex integrative function of the NTS and, potentially, other CNS structures involved in cardiovascular regulation.

\section{References}

Berger, A. J. (1979) Distribution of carotid sinus nerve afferents to solitary tract nuclei of the cat using transganglionic transport of horseradish peroxidase. Neurosci. Lett. 14: 153-158.

Ciriello, J. (1983) Brainstem projections of aortic baroreceptor afferent fibers in the rat. Neurosci. Lett. 36: 37-42.

Ciriello, J., and F. R. Calaresu (1981) Projections from buffer nerves to the nucleus of the solitary tract: An anatomical and electrophysiological study in the cat. J. Auton. Nerv. Syst. 3: 299-310.

Cowley, A. W., E. W. Quillen, and B. J. Barber (1980) Further evidence for lack of haroreceptor control of long-term level of arterial pressure. In Arterial Baroreceptors and Hypertension, P. Sleight, ed., pp. 391399, Oxford U. P., Oxford.

Crawford, J. M., and D. R. Curtis (1964) The excitation and depression of mammalian cortical neurons by amino acids. Br. J. Pharmacol. 23: 313-329.

Crill, W. E., and D. J. Reis (1968) Distribution of carotid sinus and depressor nerves in the cat brain stem. Am. J. Physiol. 214: 269-276.

Curtis, D. R., and R. W. Ryall (1966) Pharmacological studies upon spinal presynaptic fibers. Exp. Brain Res. 1: 195-204.

Dejong, W., and M. M. Palkovits (1976) Hypertension after localized transection of brainstem fibers. Life Sci. 18: 61-64.

Dejong, W., P. Fanberg, M. Palkovits, and B. Bohus (1977) Acute and chronic hypertension after lesions and transections of the rat brainstem. Prog. Brain Res. 47: 189-198.

Doba, N., and D. J. Reis (1973) Acute fulminating neurogenic hypertension produced by brainstem lesions in the rat. Circ. Res. 32: 584-593.

Engberg, I., J-A. Flatman, and J. D. C. Lambert (1979a) The actions of excitatory amino acids on motoneurons in the feline spinal cord. J. Physiol. (Lond) 228: 227-261.

Engberg, I., J-A. Flatman, and J. D. C. Lambert (1979b) A comparison of extracellular and intracellular recording during extracellular microiontophoresis. J. Neurosci. Methods 1: 219-233.

Fieglgansberger. W., and E. A. Puil (1973) Actions of glutamic acid on spinal neurons. Exp. Brain Res. 17: 35-49.
Goodchild, A. K., R. A. L. Dampney, and R. Bandler (1982) A method of cvoking physiological responses by stimulation of cell bodies, but not axons of passage, within localized regions of the central nervous system. J. Neurosci. Methods 6: 351-361.

Jordan, D., and M. K. Spyer (1977) Studies on the termination of sinus nerve afferents. Pfluegers Arch. 369: 65-73.

Kalia, M., and K. Fuxe (1985) Rat medulla oblongata. I. Cytoarchitectonic considerations. J. Comp. Neurol. 223: 285-307.

Kalia, M., and M.-M. Mesulum (1980) Brain stem projections of sensory and motor components of the vagus complex in the cat. I. Cervical vagus and nodose ganglion. J. Comp. Neurol. 211: 248-264.

Kalia, M., K. Fuxe, T. Hökfelt, O. Johannson, R. Lang, D. Ganten. C. Cuello, and L. Terenius (1984) Distribution of neuropeptide immuno-reactive nerve terminals within the subnuclei of the tractus solitarius of the rat. J. Comp. Neurol. 222: 409-444.

Locwy, A. D., E. M. Grcgorie, S. McKellar, and R. P. Baker (1979) Electrophysiological evidence that the A5 catecholamine cell group is a vasomotor center. Brain Res. 178: 196-200.

McCrimmon, D. R., J. L. Feldman, and D. F. Speck (1986) Respiratory motoneuronal activity is altered by injections of picomoles of glutamate into cat brain stem. J. Neurosci. 6: 2384-2392.

Murra, M., and D. J. Reis (1969) Termination and secondary projections of the carotid sinus nerve in the cat brainstem. Am. J. Physiol. 217: 142-153.

Nicholson, C. (1985) Diffusion from an injected volume of a substance in brain tissue with arbitrary volume fraction and tortuosity. Brain Res. 333: 325-329.

Nosaka, S., T. Yomamoto, and K. Yasunaga (1979) Localization of vagal cardioinhibitory preganglionic neurons within rat brain stem. J. Comp. Ncurol. 186: 79-82.

Panneton, W. M., and A. D. Loewy (1980) Projections of the carotid sinus nerve to the nucleus of the solitary tract in the cat. Brain Res. 191: 239-244.

Paxinos, G., and C. Watson (1986) The Rat Brain in Stereotaxic Coordinates, Academic, New York.

Ranck, J. B. (1975) Which elements are excited in electrical stimulation of mammalian central neurons system: A review. Brain Res. 98: 417-440.

Reis, D. J., A. R. Granata, M. H. Perrone, and W. T. Talman (1981) Evidence that glutamic acid is the neurotransmitter of baroreceptor afferents terminating in the nucleus tractus solitarius (NTS). J. Auton. Nerv. Syst. 3: 321-334.

Seller, H., and M. Illert (1969) The localization of the first synapse in the carotid sinus baroreceptor reflex pathway and its alteration of the afferent input. Pfluegers Arch. 306: 1-19.

Siegel, S. (1956) Nonparametric Statistics for the Behavioral Sciences, McGraw-Hill, New York.

Spyer, K. M. (1981) Neural organization and control of the baroreceptor reflex. Rev. Physiol. Biochem. Pharmacol. 88: 23-124.

Spyer, K. M., S. Donoghue, R. B. Felder, and D. Jordan (1984) Processing of afferent inputs in cardiovascular control. Clin. Exp. Hypertension A6: 173-184.

Talman, W. T., M. H. Perrone, and D. J. Reis (1980) Evidence for L-glutamate as the neurotransmitter of primary baroreceptor afferent nerve fibers. Science. 209: 813-815.

Talman, W. T., M. H. Perrone, P. Scher, S. Kwo, and D. J. Reis (1981) Antagonism of the baroreceptor reflex by glutamate diethyl ester, and antagonist to L-glutamate. Brain Res. 217: 186-191.

Talman, W. T., A. R. Granata, and D. J. Reis (1984) Glutamatergic mechanisms in the nucleus tractus solitarius in blood pressure control. Fed. Proc. 43: 39-44. 